2

\title{
Aesthetics and Entropy III. Aesthetic measures
}

\author{
M. R. V. Sahyun ${ }^{1^{*}}$ \\ 1 Consultant, Maplewood, MN USA \\ * Correspondence: sahyun@infionline.net; 1-(651)-927-9686
}

Abstract: We examined a series of real-world, pictorial photographs with varying characteristics, along with their modification by noise addition and unsharp masking. As response metrics we used three different versions of the aesthetic measure originally proposed by Birkhoff. The first aesthetic measure, which has been used in other studies, and which we used in our previous work as well, showed a preference for the least complex of the images. It provided no justification for noise addition, but did reveal enhancement on unsharp masking. Optimum level of unsharp masking varied with the image, but was predictable from the individual image's GIF compressibility. We expect this result to be useful for guiding the processing of pictorial photographic imagery. The second aesthetic measure, that of informational aesthetics based on entropy alone failed to provide useful discrimination among the images or the conditions of their modification. A third measure, derived from the concepts of entropy maximization, as well as the hypothesized preference of observers for "simpler", i.e., more compressible, images, yielded qualitatively the same results as the more traditional version of the measure. Differences among the photographs and the conditions of their modification were more clearly defined with this metric, however.

Keywords: aesthetic measure; entropy; information theory; digital photography; unsharp masking

\section{Introduction}

This article represents a continuation of studies that we undertook to see if information theory could provide a guide to the optimization of primarily pictorial images from an aesthetic point of view [1,2]. This has been a popular goal among imaging scientists [3-5]. Our approach was based on the paradigm of Kobayashi [6] who proposed that the practice of professional photographers could be a guide to automated image optimization. Thus individuals whose livelihood depend on production of images to satisfy clients, curators or exhibition jurors might in the course of their work have developed an intuitive understanding of the principles underlying the perception of their work. The ability of artists to intuitively grasp principles of perception prior to the scientific articulation of these principles is not unknown in neuroscience [7].

In the first of our studies we demonstrated that the brightness distribution embodied in some classic fine art photographs e.g., work of Julia Margaret Cameron, Robert Mapplethorpe, Edward Steichen, and Ansel Adams inter alia, did indeed correspond to a 
Shannon entropy, S, maximum for the images. In the second study, exploring the effects of variables commonly manipulated by professional photographers, namely unsharp masking and noise addition, we concluded that maximum entropy alone did not provide a sufficient figure of merit to guide, for example, automated image optimization, but we found a more promising response metric in Birkhoff's aesthetic measure, $M,[8]$. The aesthetic measure as originally formulated by Birkhoff is the ratio of order to complexity. Since entropy, $S$, is commonly understood as a measure of disorder, this original formulatioin iscontrary to the expectation that maximum entropy imagery is most desirable $[1,3,5]$. Accordingly in our previous work we used the aesthetic measure as adapted by Bense [9] and Gauvrit [10],

$$
M=(n S-K) / n S \text {; }
$$

$n$ is a normalization constant, and $K$ is a measure of image compressibility. We have understood $\mathrm{S}$ as Gibbs entropy,

$$
S=-k \sum \ln [p(x)]
$$

where $p(x)$ is the probability that a given pixel corresponds to the brightness level, $x$. Note that Gibbs entropy differs from Shannon entropy in that the former uses logarithms to base $e$, while the latter uses base two. The use of the aesthetic measure as expressed on the form of Eq. (1) in the present context is also consistent with the conclusion of Gauvrit and co-workers [10], namely that people show a preference for high image entropy but low algorithmic complexity, i.e., a more simply discernible internal logic to an information rich image.

In the context of understanding the visualization of an image as a product of the creative process the aesthetic measure has been understood as the ratio between algorithmic "reduction of uncertainty" (internal logic of the image) and complexity according to Rigau and co-workers [11]. These authors have attempted to define the aesthetic measure exclusively in terms of entropy. Thus

$$
M^{\prime}=1-S / S \max ,
$$

whereby it is assumed that entropy can function as a measure of complexity on the basis of the Shannon source coding theorem. This approach is known as "informational aesthetics".

There have been a number of other approaches to the quantification of aesthetic response; these have been reviewed critically by Burns [12], who has proposed his own so-called EVE' model. The critical advance in Burns' approach is the recognition that aesthetic response may vary among individuals, which makes the assignment of any single aesthetic figure-of-merit to an image problematical. The EVE' approach will not be taken in the present work, however, as it seems primarily appropriate in conjunction with psychometric studies. It makes use of a concept called "visual entropy", application of which to simple, abstract patterns is straightforward, but whose application to complex real-world photographic images seems problematical.

According to Eysenck [13] aesthetic response can be expressed as the product of order and complexity, $K / S$, in the present notation, insofar as entropy reflects disorder and is the inverse of order as originally understood by Birkhoff [8]. This approach essentially equates aesthetic attractiveness with complexity, since both entropy and the compression 
factor reflect complexity. Eysenck's understanding is not supported by the work of Gauvrit [10], Burns [12] or Berlyne [14]. In the present work we propose another formulation of the aesthetic measure, $M^{\prime \prime}$, essentially the inverse of Eysenck's proposal; thus

$$
M^{\prime \prime}=n S / K \text {. }
$$

This formulation incorporates both the desirability of high image entropy, as inferred from imaging science [1,3-5] and the preference for algorithmically simpler, i.e., more compressible, presentations [10].

Also we continue to look at the aesthetic and entropic consequences of digital image modification by noise addition, in keeping with the philosophy of Kobayashi and co-workers [6]. Both of these artifices are commonly employed by professional photographers [15]. It has been proposed that noise addition specifically raises the contrast threshold for pattern detection [16], allowing the viewer to ignore lower contrast, distracting detail, so-called "entropy masking". An additional rationale for considering unsharp masking as a variable in image optimization was the understanding that it is one of the image processing steps which occur in the human retina prior to encoding visual information for transmission by the optic nerve $[17,18]$. An appreciation of what level of unsharp masking provided the optimum contribution to image perception might therefore serve as a guide for the image processing to be incorporated in retinal prosthetic devices [19].

Our previous studies [1,2] suffered from two shortcomings: (1) a limited number of images were evaluated, so the generality of the conclusions was problematical; (2) the aesthetic measure was parameterized using JPEG compressibility to evaluate $K$. More recent psychophysical studies have shown GIF compressibility to correlate better with perceived image attractiveness, i.e., "beauty". In this psychophysical study it was inferred that GIF compressibility provides the best automated estimate of algorithmic complexity, i.e., Kolmorgorov comoplexity, of the image [11]. The purpose of the present extension of our work is therefore threefold:

- to extend our studies to a greater range of image types;

- to re-evaluate the use of the aesthetic measure applied to photographic imagery using GIF compressibility; and

- to compare the approaches to aesthetic measure embodied in Eqs. (3) and (4).

\section{Methodology}

Flve photographs of disparate characteristics were selected for the study, as shown in Figure 1:

- Flag is a relatively simple, high contrast image with strong linear elements;

- Oak Park is a visually complex landscape photograph, exhibiting considerable chiaroscuro; it had previously been shown to exhibit a nearly Gaussian brightness distribution which approximated a maximum entropy ideal [1];

- Author was selected as representative of typical casual consumer portraits.

- Rocks is a nearly abstract "pattern" image with no clearly definable center of interest; 
124

125

126

127

(a)

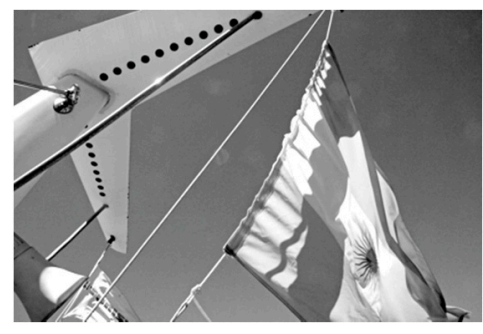

(b)

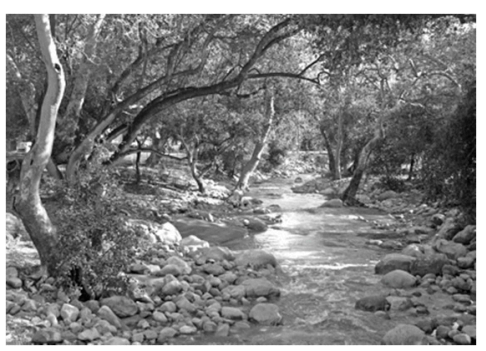

(c)

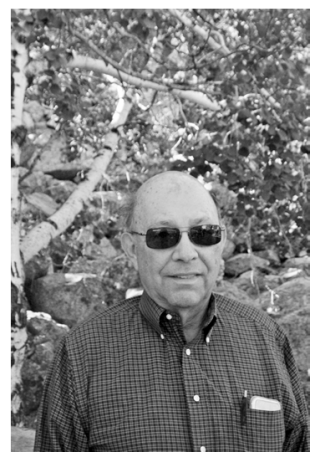

128

129

130

131

132

133

134

135

136

137

138

139

140

141

142

143

144

145

146

147

148

149

150
- Naval Hero is a photograph of a statue, which, like Oak Park, exhibits strong chiaroscuro and a visually complex background. 
151

152

153

154

155

156

157

158

159

160

161

162

$$
K=G I F / B M P
$$

where the bitmap file sizes are 30MB for Flag, Rocks and Naval Hero, 22MB for Oak Park, and $8.8 \mathrm{MB}$ for Author, which had been cropped by the photographer. The use of degree of compression achieved by a real-world algorithm has been proposed to by a useful approximation of Kolmorgorov complexity, i.e., the computational resources needed to specify the image, by Rigau and co-workers [10].

\section{Results and Discussion}

\subsection{Entropy}

The entropies calculated according to Eq. (5) for each of the images with their various modifications are given in Table 1.

Table I.-Image Entropy, $S / k$.

\begin{tabular}{|c|l|l|l|l|l|}
\hline & Flag & Rocks & Author & Naval Hero & Oak Park \\
\hline As taken & 1574 & 1591 & 1496 & 1537 & 1514 \\
\hline Noise addition: & & & & & \\
\hline $10 \%$ & 1584 & 1591 & 1466 & 1541 & 1505 \\
\hline $20 \%$ & 1540 & 1587 & 1435 & 1536 & 1488 \\
\hline $50 \%$ & 1484 & 1562 & 1402 & 1526 & 1460 \\
\hline $100 \%$ & 1460 & 1491 & 1382 & 1473 & 1433 \\
\hline Unsharp masking: & & & & & \\
\hline $25 \%$ & 1567 & 1578 & 1470 & 1529 & 1488 \\
\hline $50 \%$ & 1560 & 1565 & 1443 & 1545 & 1460 \\
\hline $100 \%$ & 1559 & 1553 & 1449 & 1529 & 1475 \\
\hline $200 \%$ & 1571 & 1524 & 1415 & 1521 & 1470 \\
\hline $500 \%$ & 1574 & --- & 1394 & 1502 & 1441 \\
\hline
\end{tabular}

163

164

165

166

167

168

169

170

171

172

173

174

175

176

It can be seen from these data that neither noise addition nor unsharp masking result in substantial changes in the entropy of any of the images. There is a small monotonic decrease in entropy with increasing noise addition for all the images that becomes significant at 50 and $100 \%$ noise addition. It is of course expected that the addition of noise should affect image entropy as

$$
(S / k) \text { total }=[(S / k) \text { image }+b(S / k) \text { noise }] /(1+b)
$$

where $b(=\%$ Noise $/ 100)$ is a coefficient reflecting the level at which noise has been added. The expected value of $(S / k)$ noise is $1420 \mathrm{~S} / \mathrm{k}$ units [cf. Eq. (9) below]. Correlation of the predictions of Eq. (6) with the data of Table 1 yield a correlation coefficient, $r=0.916$. To confirm that the monochrome noise filter in the version of Photoshop Elements ${ }^{\mathrm{TM}}$ used in this work introduced white noise, an image comprising noise alone at the $100 \%$ level was created; its histogram is shown in Figure 2. 


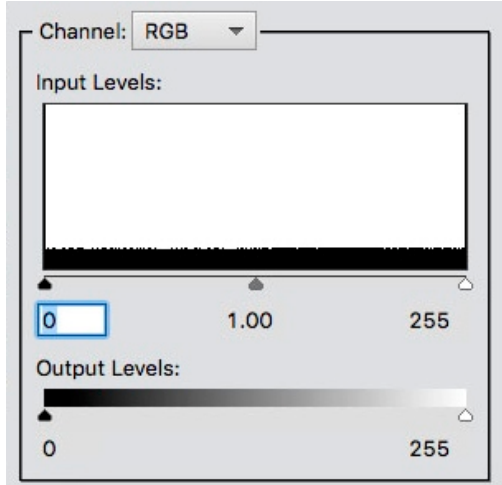

Figure 2.- Histogram for white noise generated by the noise filter in Photoshop Elements $^{\mathrm{TM}} 11$.

The magnitudes of the changes in entropy with unshahrp masking are, however, very image dependent. Because of its insensitivity to the image modifications employed, even at high levels, we infer that entropy alone is probably not a useful metric to employ alone in image optimization, reinforcing the conclusion from our preliminary investigation [2].

It should be obvious from the data of Table 1 that entropy is not a sufficient measure of information content of an image. Entropy calculated according to Eq. (5) does not reflect the resolution, i.e., information density, of the image insofar as the number of pixels is significantly greater than the number of brightness levels, 256 in the present case. As all the images used in the present work were captured using the same device, this caveat is not relevant to the inferences drawn in this study.

\subsection{Compressibility and complexity}

Significant changes in GIF compressibility result on noise addition and/or unsharp masking, and these again vary with the different types of images. (Table 2). It should be noted in this connection that the GIF file format, although it uses the Lempel-Ziv-Welch (LZW) lossless data compression technique [20], does not lead to lossless compression. Inspection of the histograms of the images saved in this format reveal that compression is achieved in part by reduction of the number of brightness levels, as shown in Figure 3 for the Flag image, just as GIF compression reduces the color gamut of RGB imagery [20]. The number of levels may be reduced by up to about $44 \%$ for the images studied here, i.e., to ca. 144 levels from the 256 levels used in recording the image. This in turn leads to a ca. $50 \%$ reduction in the information content, as entropy, of the images.

Table 2.-Image compressibility factor, $K=G I F / B M P$.

\begin{tabular}{|c|l|l|l|l|l|}
\hline & Flag & Rocks & Author & Naval Hero & Oak Park \\
\hline As taken: & 0.233 & 0.267 & 0.307 & 0.320 & 0.373 \\
\hline Noise addition: & & & & & \\
\hline $10 \%$ & 0.323 & 0.340 & 0.386 & 0.340 & 0.355 \\
\hline $20 \%$ & 0.373 & 0.376 & 0.386 & 0.380 & 0.382 \\
\hline $50 \%$ & 0.387 & 0.370 & 0.386 & 0.357 & 0.373 \\
\hline $100 \%$ & 0.277 & 0.274 & 0.420 & 0.277 & 0.350 \\
\hline
\end{tabular}


205

206

207

\begin{tabular}{|c|l|l|l|l|l|}
\hline Unsharp Masking: & & & & & \\
\hline $25 \%$ & 0.210 & 0.277 & 0.295 & 0.276 & 0.345 \\
\hline $50 \%$ & 0.217 & 0.287 & 0.273 & 0.270 & 0.323 \\
\hline $100 \%$ & 0.256 & 0.307 & 0.295 & 0.283 & 0.336 \\
\hline $200 \%$ & 0.320 & 0.327 & 0.318 & 0.303 & 0.305 \\
\hline $500 \%$ & 0.300 & --- & 0.341 & 0.317 & 0.314 \\
\hline
\end{tabular}

(a)

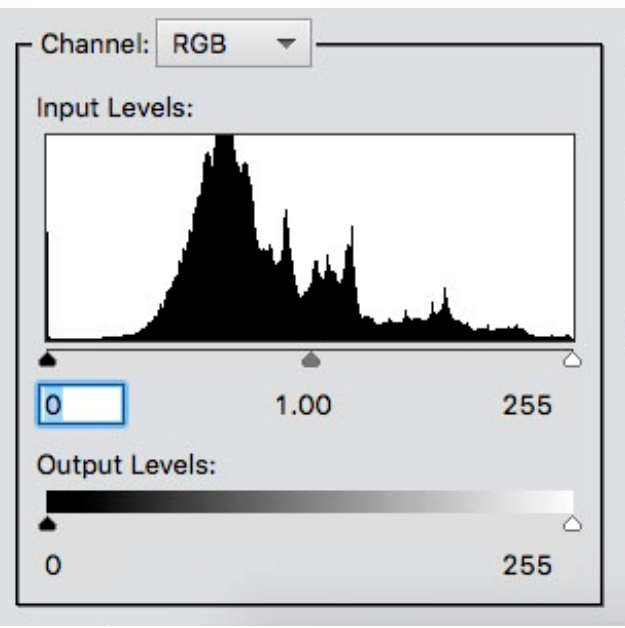

(b)

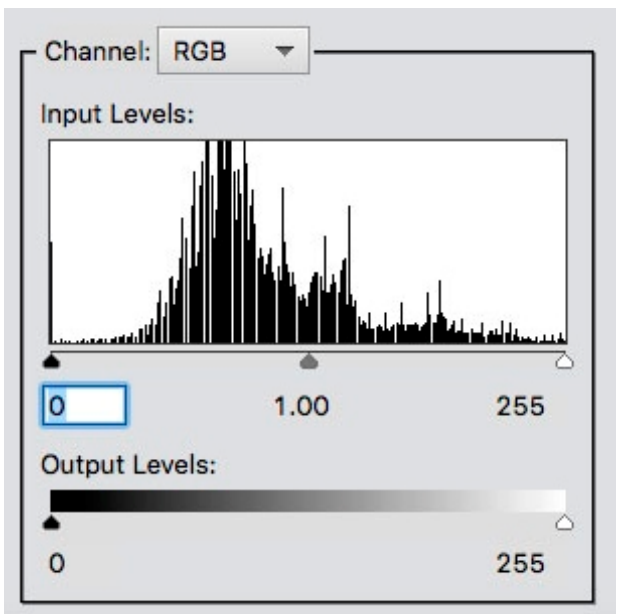

Figure 3.-Brightness distributions for Flag image (a) as taken, and (b) after GIF compression.

Previously we suggested the desirability of a perceptually based metric of compressibility [2]. A starting point for this effort might be the light-adaptation of the HVS as expressed in the Weber's law coefficients for rod and cone vision, which in turn define the number of "just-noticeable-difference" (JND) increments perceivable over the range of loglinear response of the HVS [21]. Subsequent research has shown that these JND increments are image dependent, as well as dependent on the display device and the viewing conditions [22]. GIF compressibility may correlate successfully with perceived aesthetic quality of the image precisely because this compression algorithm drops out brightness levels in the image to create gaps in the brightness distribution function that do not exceed the JND increments distinguishable by a lay observer under typical viewing conditions.

The compression factors, $K$, realized for the five unmodified images are consistent with the visual impression of the images, namely that Flag is the least complex while Oak Park is the most complex. Noise addition reduces the GIF compressibility of the relatively simple Flag image, Rocks, and of the casual portrait, Author, much more than for Naval Hero and the relatively complex landscape image, Oak Park, whose compressibility is relatively unaffected by noise addition. There is in fact a negative correlation between the maximum increase in the compression factor (decrease in compressibility), $\Delta K$, and $K$ for the unmodified image $(r=0.993)$,

$$
\Delta K=0.37-0.97 K \text {, }
$$


231 which means that the most compressible images exhibit the most change in 232 compressibility on noise addition. Unsharp masking produces opposite effects on the 233 compressibility of Flag and Rocks on the one hand, which exhibit decreased 234 compressibility (increased $K$ ) on unsharp masking, and the more complex Naval Hero and 235 Oak Park, which exhibit increased compressibility (decreased $K$ ) on the other. There is 236 little significant effect on the compressibility of Author.

237

238

239

240

241

242

243

244

245

246

247

248

249

250

251

252

253

254

\subsection{Aesthetic measure, $M$, from Eq. (1)}

The aesthetic measure, $M$, was calculated from these data according to Eq. (1) with a normalization factor $n=0.000705$, as the reciprocal of the reduced entropy,

$S \max / k$, for a fully equalized 256 level brightness histogram, proposed $[3,23]$ to correspond to a maximum entropy case, i.e.,

$$
n=k / S \max =[256 \ln (256)]^{-1} \text {. }
$$

The compression factor, $K$, was estimated as described above in Eq. (3) from the GIF file size (Table 2). Data for $M$ are given in Table 3, from which it is apparent that the aesthetic measure for the images as taken stands in inverse relationship to algorithmic complexity. The variation in $M$ is small but, given an expected uncertainty of ca. \pm 0.005 in the values, real. This relationship is as expected from Eq (1) and the interpretation of Gauvrit [10], but inconsistent with the expectations of Berlyne who posited a preference for an intermediate level of complexity [14]. Ithough the value of $M$ is very sensitive to the value chosen for $n$, there is so little variation in image entropy resulting in these experiments that variation in the aesthetic measure $M$ is dominated by variation in $K$.

Table 3.-Birkhoff aesthetic measure, $M$, from Eq. (1).

\begin{tabular}{|c|l|l|l|l|l|}
\hline & Flag & Rocks & Author & Naval Hero & Oak Park \\
\hline As taken: & 0.788 & 0.760 & 0.707 & 0.702 & 0.648 \\
\hline Noise addition: & & & & & \\
\hline $10 \%$ & 0.708 & 0.695 & 0.623 & 0.685 & 0.663 \\
\hline $20 \%$ & 0.656 & 0.661 & 0.615 & 0.646 & 0.633 \\
\hline $50 \%$ & 0.628 & 0.662 & 0.606 & 0.666 & 0.631 \\
\hline $100 \%$ & 0.729 & 0.737 & 0.565 & 0.735 & 0.651 \\
\hline Unsharp Masking: & & & & & \\
\hline $25 \%$ & 0.808 & 0.749 & 0.713 & 0.741 & 0.668 \\
\hline $50 \%$ & 0.801 & 0.737 & 0.730 & 0.750 & 0.685 \\
\hline $100 \%$ & 0.764 & 0.717 & 0.709 & 0.735 & 0.674 \\
\hline $200 \%$ & 0.709 & 0.694 & 0.679 & 0.715 & 0.704 \\
\hline $500 \%$ & 0.728 & --- & 0.650 & 0.699 & 0.689 \\
\hline
\end{tabular}

It can be seen that among the image modifications investigated, small improvements in aesthetic measure are achieved by implementation of unsharp masking four of the five images. For the simplest image, Flag, the maximum improvement is achieved at only $25 \%$ unsharp masking. The improvement is largest for the more complex Oak Park image but in 
261

262

263

264

265

266

267

268

269

270

271

272

273

274

275

276

277

278

279

280

281

282

283

284

285

286

287

288

289

masking employed, with a maximum at the $200 \%$ level. The visual effect on this image of unsharp masking at this level is shown in Figure 4.

(a)

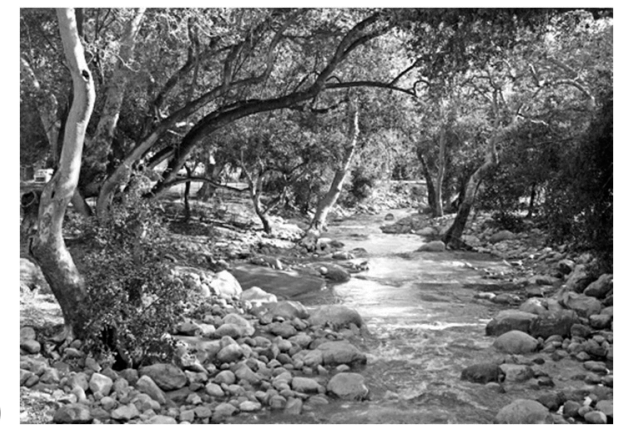

(b)

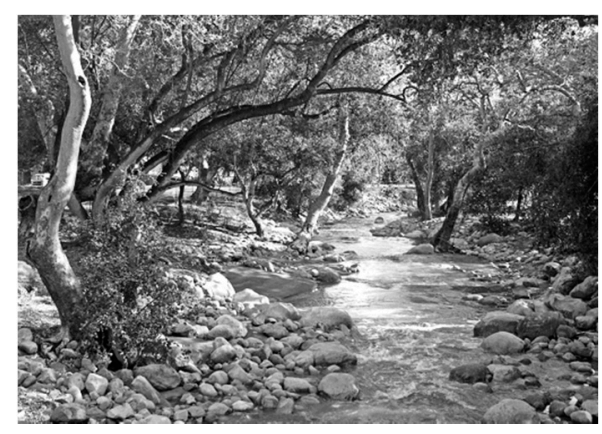

(c)

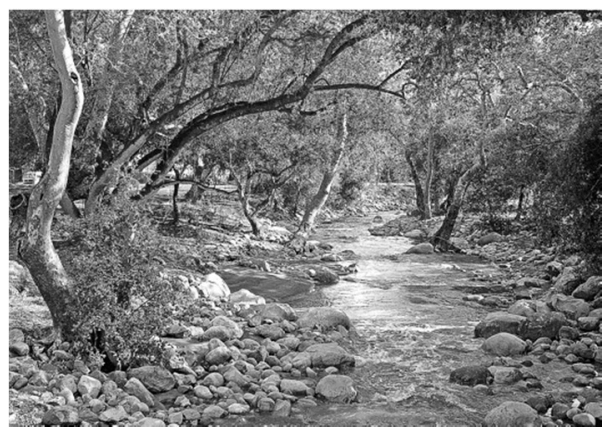

Figure 4.-Oak Park image: (a) as taken; (b) with 50\% unshahrp masking; (c) with $200 \%$ unsharp masking.

The data suggest a loglinear relationship between an image's algorithmic complexity, as measured by $K$, and the level of unsharp masking, \%USmax, yielding a maximum in aesthetic measure $(r=0.915)$ :

$\ln (\%$ USmax $)=9.1+4.3 \ln K$.

(For this calculation \%US for the Rocks image was taken as $25 \%$, the level which exhibited the minimum decrease in aesthetic measure). In general we infer that the greater an image's algorithmic complexity, the higher the level of unsharp masking appropriate to maximize the image's aesthetic appeal, i.e., to maximize its perceptual simplification, as the aesthetic measure metric is dominated by the compressibility factor in Eq. (1). Accordingly Eq. (10) provides a guide for the application of unsharp masking by practical photographers as well as the basis for an automatic selection of the level of unsharp masking appropriate for a given image.

The limited data set, with the exception of Rocks, which showed no improvement in aesthetic measure under any of the modification conditions, also suggests a reciprocal relationship between GIF compressibility of the unmodified image and the optimal improvement in aesthetic measure achievable by unsharp masking, $\Delta M \max (r=0.844)$, The decrease in $M$ at higher levels of unsharp masking for the Flag and Author images may be attributed in part to the enhanced perceived graininess of these images with these higher levels. Unsharp masking at the radius employed effectively adds noise, along with some posterization and artifact enhancement, to the images, as is shown in Figure 5 of the Flag image with $500 \%$ unsharp masking. 
Figure 5.- Flag image with 500\% unsharp masking.

Deliberate noise addition led to negative $M$ for both of these images along with the Rocks image, while being of little consequence to the aesthetic measure for the more complex Oak Park and Naval Hero images. Any increase in the contrast threshold for pattern detection, as hypothesized by Watson et al. [16] does not appear to be reflected in the aesthetic measure data.

\subsection{Aesthetic measure, $M^{\prime}$, from Eq. (3)}

Informational aesthetic measures, $M^{\prime}$, were calculated for each image and each modification according to Eq. (3) combined with Eq. (9), so that $M^{\prime}=1-n S / k$. Data are reported in Table 4. It can be seen that the values of $M^{\prime}$ are clustered about a central tendency value of zero $( \pm 0.037)$.

Table 4.- Entropy based aesthetic measure, $M^{\prime}$, from Eq. (2).

\begin{tabular}{|c|l|l|l|l|l|}
\hline & Flag & Rocks & Author & Naval Hero & Oak Park \\
\hline As taken: & -0.036 & -0.047 & 0.015 & -0.012 & 0.003 \\
\hline Noise addition: & & & & & \\
\hline $10 \%$ & -0.043 & -0.047 & 0.036 & -0.014 & 0.009 \\
\hline $20 \%$ & -0.014 & -0.045 & 0.055 & -0.011 & 0.020 \\
\hline $50 \%$ & 0.023 & -0.028 & 0.077 & -0.004 & 0.038 \\
\hline $100 \%$ & 0.039 & 0.018 & 0.090 & 0.030 & 0.057 \\
\hline Unsharp Masking: & & & & & \\
\hline $25 \%$ & -0.032 & -0.039 & 0.032 & -0.006 & 0.020 \\
\hline $50 \%$ & -0.027 & -0.030 & 0.050 & -0.017 & 0.037 \\
\hline $100 \%$ & -0.026 & -0.022 & 0.046 & -0.006 & 0.029 \\
\hline $200 \%$ & -0.034 & -0.003 & 0.068 & -0.001 & 0.032 \\
\hline $500 \%$ & -0.036 & --- & 0.083 & 0.012 & 0.051 \\
\hline
\end{tabular}

The data provide little basis for discrimination among the images, as captured, on the basis of aesthetic value, even though it is reasonable to expect significant differences in viewer preference among the five images. Statistically significant values of $M^{\prime}\left(\left|M^{\prime}\right| \geq 0.05\right.$, based on the estimated reproducibility of the entropy estimates, above) are obtained 
320

321

322

323

324

325

326

only for the Author at the higher levels of noise addition and for Oak Park with 100\% noise addition. [Note that for the case of Author, the estimated entropy, $S / k=1382$, is significantly different from the prediction of Eq. (7), $S / k=1458$, for which $M^{\prime}=$ -0.0276, within experimental error of zero]. Visual inspection of the imagery suggests that this highest level of noise addition is well above that which can enhance feature detection by entropy masking [16]. See Figure 6.

(a)

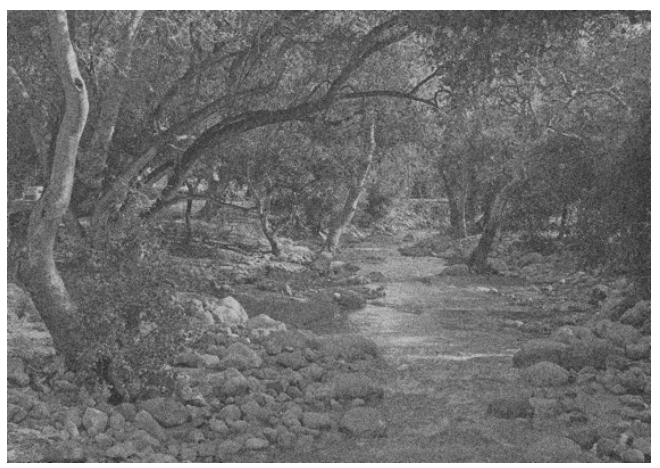

(b)

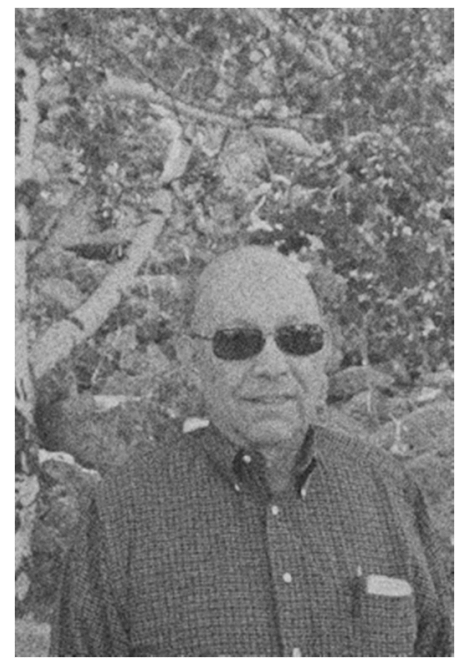

Figure 6.- Test images with $100 \%$ noise addition: (a) Oak Park; (b) Author.

Unsharp masking yields significant positive values of $M^{\prime}$ only for the Author image at $200 \%$ and 500\% unsharp masking, and for Oak Park at 500\% unsharp masking, above the normally useful level, insofar as it introduces significant noise through grain enhancement as well as artifact introduction (see above). The Author image with $500 \%$ unsharp masking is shown in Figure 7. Degradations introduced by this level of unsharp masking are not as visually apparent as they were in the Flag image (Fig. 5), and the resulting image might be deemed at least acceptable.

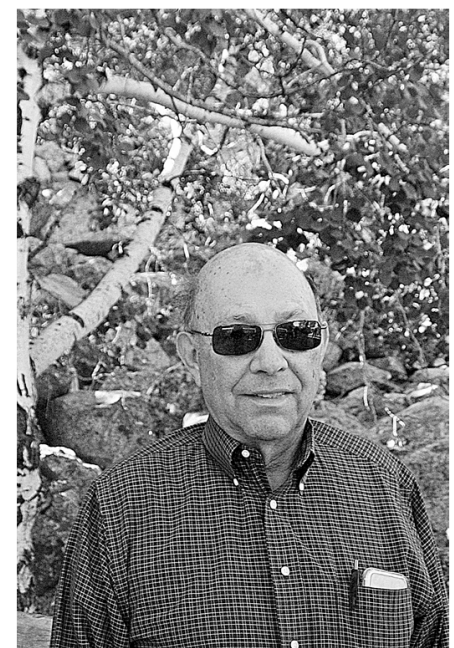

Figure 7.-Author image at $500 \%$ unsharp masking. 
In our previous study [2] we inferred a disconnect between entropy and compressibility despite expectations from the Shannon source coding theorem, on which Eq. (3) is based. This disconnect is apparent from comparison of the data of Tables 1 and 2. While entropy may reflect some aspects of complexity, it apparently does not reflect others, which may be important to the observer's experience. This inference is consistent with the existence of complexity in a multidimensional space as discussed by Nadal and co-workers [24].

Eq. (3) is intrinsically biased to return more positive aesthetic measures when images are modified so as to reduce their information content as measured by Shannon entropy, i.e., on differentiation of Eq. (3), $\partial M^{\prime} / \partial S<0$. This relationship has been described as a "...preference for minimalist structures" [25]. It is contrary to the implication from the study of Singnoo and Finlayson [5] who found that viewers tended to optimize image contrast to a level corresponding to an entropy maximum. The use of Eq. (3) and the fundamental idea of informational aesthetics, namely that an aesthetic measure, which is taken as a ratio (or difference) between information content and complexity, can be expressed exclusively in terms of entropy has been criticized by Burns [12] who concludes that "...classical entropy is not...an adequate measure of visual complexity." The inability of Eq (2) to distinguish among the five disparate test images and the various modifications employed in this study supports Burns' inference.

Previous work on the use of various aesthetic measures has focused on their application to simple abstract images, e.g., Mondrians [12]. In the work of Rigau et al. in which the informational aesthetic measure, equivalent to Eq. (3), was proposed, the most significant positive aesthetic measures were obtained for Mondrians, compared to more representational, pictorial imagery, e.g., paintings by Van Gogh [10]. Accordingly we infer that approaches to quantifying aesthetic properties that have been derived with respect to simple abstract images may not be relevant to the more complex imagery characteristic of the real world, especially high resolution pictorial photographs.

\subsection{Aesthetic measure, $M^{\prime \prime}$, from Eq. (4)}

Aesthetic measures, M", calculated according to Eq. (4) are given in Table 5. The magnitudes of these response metrics are much greater than those obtained by use of Eq. (3) in Table 3. The aesthetic measure in the latter case is represented on a zero-to-one scale, while the scale of Eq. (4) is open-ended. Because of its greater scale, Eq. (4) appears to provide a more sensitive response metric. We estimate the uncertainty in the estimates reported in Table 5 to be ca. $\pm 0.10 ; M^{\prime \prime}$ is therefore reported to only two significant figures. This formulation of the aesthetic measure is also more sensitive to entropy variations than the modification of Birkhoff's expression embodied in Eq. (1). From Eq. (4) $\partial M^{\prime \prime} / \partial S=n / K$, while for Eq. (1) $\partial M / \partial S=K / n S^{2}$; in the present study (data from Table 1) $n S$ is approximately unity. Thus $\partial M / \partial S=n K$, where $K$ is by definition less than unity. Given the values of $K$ observed for our test images (Table 2), the numerical value of $\partial M^{\prime \prime} / \partial S$ [Eq. (4)] may be an order of magnitude larger than that of $\partial M / \partial S$ [Eq. (1)]. 
Table 5.-Aesthetic measure, $M^{\prime \prime}$, from Eq. (3).

385

\begin{tabular}{|c|l|l|l|l|l|}
\hline & Flag & Rocks & Author & Naval Hero & Oak Park \\
\hline As taken: & 4.8 & 4.2 & 3.4 & 3.4 & 2.8 \\
\hline Noise addition: & & & & & \\
\hline $10 \%$ & 3.4 & 3.3 & 2.6 & 3.2 & 3.0 \\
\hline $20 \%$ & 2.9 & 3.0 & 2.6 & 2.8 & 2.7 \\
\hline $50 \%$ & 2.7 & 3.0 & 2.5 & 3.0 & 2.7 \\
\hline $100 \%$ & 3.7 & 3.8 & 2.3 & 3.8 & 2.9 \\
\hline Unsharp Masking: & & & & & \\
\hline $25 \%$ & 5.2 & 4.0 & 3.5 & 3.9 & 3.0 \\
\hline $50 \%$ & 5.0 & 3.8 & 3.7 & 4.0 & 3.2 \\
\hline $100 \%$ & 4.2 & 3.5 & 3.4 & 3.8 & 3.1 \\
\hline $200 \%$ & 3.4 & 3.3 & 3.1 & 3.5 & 3.4 \\
\hline $500 \%$ & 3.7 & --- & 2.9 & 3.3 & 3.2 \\
\hline
\end{tabular}

386

However, comparing Tables 3 and 5, we see that the qualitative trends are the same for both $M$ and $M^{\prime \prime}$. Among the photographs as taken, Flag has the largest aesthetic measure $M^{\prime \prime}$, and Oak Park has the lowest, again predicting a preference for the simplest, least algorithmically complex image. Noise addition, with the exception of one data point, Oak Park with $10 \%$ noise, is predicted to be detrimental to the attractiveness of the image. At the same time, some enhancement of the aesthetic measure results from unsharp masking, with the maximum values of $M^{\prime \prime}$ obtained at levels of unsharp masking, \%USmax, that, as with the values of $M$, the aesthetic measure of Eq (1), vary with the image. These preferred levels of unsharp masking correspond to the same levels indicated by $M$; Eq. (10) still applies. Thus for the specific application to pictorial photographs, which tend to be high entropy images which undergo only small entropy changes with variation in subject matter or on modification, we prefer the aesthetic measure formulation embodied in Eq. (4) to some of the formulations based largely on studies involving simple abstract patterns [10-13].

\section{Conclusions}

We examined a series of real-world, pictorial photographs with varying characteristics, along with their modification by noise addition and unsharp masking. Both techniques are common in the practice of photography. As response metrics we used three different versions of the aesthetic measure, originally proposed by Birkhoff. Gibbs entropy was evaluated for each image at each condition of modification, but exhibited little variation. The first aesthetic measure, which has been used in other studies and which we used in our previous work as well, showed a preference for the least complex of the images. It provided no justification for noise addition, but did reveal enhancement on unsharp masking. Optimum level of unsharp masking varied with the image, but was predictable from the individual image's GIF compressibility. We expect this result to be useful for 
412

413

414

415

416

417

418

419

420

421

422

423

424

425

426

427

428

429

430

431

432

433

434

435

436

437

438

439

440

441

442

443

444

445

446

447

448

449

450

451

452

453

454

guiding the processing of pictorial photographic imagery. The second aesthetic measure, that of informational aesthetics, is based on entropy alone; it failed to provide useful discrimination among the images or the conditions of their modification. A third measure, derived from the concepts of entropy maximization, as well as the hypothesized preference of observers for "simpler", i.e., more compressible, images, yielded qualitatively the same results as the more traditional version of the measure. Differences among the photographs and the conditions of their modification were more clearly defined with this metric, however.

Acknowledgments: This study was self-funded. No conflicts of interest are envisioned. We thank Irene Nordquist Sahyun for the use of the test images, to which she retains copyright.

\section{References}

1. Sahyun, M. R. V. Aesthetics and entropy: optimization of the brightness distribution. Proc. SPIE 2011, 8291, 82911H, DOI: 10.1117/12.903638.

2. Sahyun, M. R. V. Aesthetics and entropy II: a critical examination., Proc. SPIE 2013, 8651, 86511K, DOI: 10.1117/12.2019098.

3. Agaian, S.; Silver, B.; Panetta, K. A. Transform Coefficient Histogram-Based Image Enhancement Algorithms Using Contrast Entropy. IEEE Trans. Image Proc. 2007, 16(3), 741-758.

4. Panetta, K. A.; Wharton, E. J.; Agaian, S. S. Human Visual System-Based Image Enhancement and Logarithmic Contrast Measure. IEEE Trans. Systems, Man, and Cyber., B: Cybernetics 2008, 38(1), 174-188.

5. Singnoo, Jakkarin; Finlayson, G. D. Understanding the Gamma Adjustment of Images. Proc. IS\&T/SID $18^{\text {th }}$ Color Imaging Conf., Scottsdale, AZ, USA; IS\&T: Springfield, VA, 2010, 134-139; ISSN 2166-9635.

6. Kurihara, Takehito; Manabe, Yoshitsugu; Aoki, Naokazu; Kobayashi, Hiroyuki. Digital image improvement by adding noise: an example from a professional photographer. J. Imaging Sci. Technol., 2011, 55, 30503-1 - 30503-9.

7. Lehrer, J. Proust was a Neuroscientist; Houghton Mifflin Co.: New York, NY, 2007; ISBN-13: 978-0547085906.

8. Birkhoff, G. D. Aesthetic Measure; Harvard University Press: Cambridge, UK, 1933; ISBN 9780674734470.

9. Bense, M. Einführung in die informationstheoretische Ästhetik. Grundlegung und Anwendung in der Texttheorie; Rowohlt Taschenbuch Verlag $\mathrm{GmbH}$.: Reinbek-bei-Hamburg, Germany, 1969.

10. Rigau, J.; Feixas, M.; Sbert, M. Conceptualizing Birkhoff's Aesthetic Measure Using Shannon Entropy and Kolmogorov Complexity. In Computational Aesthetics in Graphics, Visualization, and Imaging; Cunningham, D. W.; Meyer, G.; Neumann, L., Eds.; Eurographics Assoc., Aire-la-Ville, Switzerland, 2007. Available online: http://imae.udg.edu/ rigau/Publications/Rigau07B.pdf (accessed 12 December 2017). 
455

456

457

458

459

460

461

462

463

464

465

466

467

468

469

470

471

472

473

474

475

476

477

478

479

480

481

482

483

484

485

486

487

488

489

490
11. Gauvrit, N.; Soler-Toscano, F.; Guida, A. A preference for some types of complexity; comment on "Perceived beauty of random texture patterns: a preference for complexity". Acta Psych. 2017, 174, 48-53.

12. Burns, K. Entropy and optimality in abstract art: an empirical test of visual aesthetics. J. Math. Arts 2015, 10, 77-90; DOI: 10.1080/17513472.2015.1096738.

13. Eysenck, H. An experimental study of the "good Gestalt"-A new approach. Psychol. Rev. 1942, 49, 334-344.

14. Berlyne, D. Complexity and incongruity variables as determinants of exploratory choice and evaluative ratings. Can. J. Psych. 1963, 17, 274-290.

15. 1x.com. Photo Inspiration; John Wiley \& Sons,: Chichester, UK, 2012; ISBN-13: 978-1118290521

16. Watson, A. B.; Borthwick, R.; Taylor, M. Image quality and entropy masking. Proc. SPIE, 1997, 3016, 1-11, DOI: 10.1117/12.274501.

17. Mead, C. A.; Mahowald, M. A silicon model of early visual processing. Neural Networks 1988, 1, 91-97, DOI: 10.1016/0893-6080(88)90024-X. Available online: https://authors.library.caltech.edu/53151/1/388888.pdf (accessed 12 December 2017).

18. Marr, D.; Hildreth, E. Theory of edge detection. Proc. Roy. Soc., Ser. B 1980, 207, 187-217.

19. Dagnelie, G. Retinal implants: emergence of a multidisciplinary field. Curr. Opin. Neurol. 2012, 25(1), 67-75, DOI: 10.1097/WCO.0b013e32834f02c3.

20. GIF. Available online: https://en.wikipedia.org/wiki/GIF (accessed 13 December 2017).

21. Kalloniatis, M.; Luu, C. Light and Dark Adaptation. Available online: http://webvision.med.utah.edu/book/part-viii-gabac-receptors/light-and-dark-ada ptation/ (accessed 14 December 2017).

22. Yang, H.; Liu, L.; Tang, H.; Qin, S.; Yin, H.; Tu, Y.; Heyndericks, I. Relationship of Just Noticeable Difference (JND) in Black Level and White Level With Image Content. J. Display Tech. 2014, 10, 470-477; DOI: 10.1109/JDT.2014.2302585.

23. Bassiou, N.; Kotropoulos, C. Color image histogram equalization by absolute discounting back-off. Comp. Vision Image Understanding 2007, 107, 108-122.

24. Nadal, M.; Munar, E.; Marty, G.; Cela-Conde, C. J. Visual Complexity and Beauty Appreciation: Explaining the Divergence of Results. Emp. Studies Arts 2010, 28, 173-191; DOI: 10.2190/EM.28.2.d.

25. Scha, R.; Bod, R. Computationele esthetica. Infor. Informatie. 1993, 11, 54-63, quoted in Ref. [12]. 